\title{
Cell Microarrays: An Emerging Technology for the Characterization of Antibodies
}

\author{
Jochen M. Schwenk, Dieter Stoll, Markus F. Templin, and Thomas O. Joos \\ NMI-Natural and Medical Sciences Institute at the University of Tübingen, Reutlingen, Germany
}

BioTechniques 33:S54-S61 (December 2002)

\section{ABSTRACT}

The possibility to miniaturize and parallelize biological assays has a great impact on the development of biomedical technologies. Here, we describe a simple, miniaturized, and parallelized method employing entire cells from different cell lines displaying a protein of interest on their surface, which were immobilized on a microarray slide. Antibodies were added to these cellular microarrays, and their specific binding to the cell surface proteins was monitored using appropriate fluorescently labeled detection molecules. This new method is applicable for rapidly screening cell surface-specific antibodies with respect to selectivity and cross-reactivity.

\section{INTRODUCTION}

DNA chips have become an established technology. Their application for screening single nucleotide polymorphisms (SNPs) and for generating transcriptional profiles of genes at a genome-wide level has led to a wealth of new insights (22). Moreover, DNA chips have gained a key position in medical and biological research and will certainly lead to new analytical approaches in our attempt to understand the inherent complexity of biological systems (14).

In the post-genomic era, the great demand for enabling technologies presents an enormous challenge for researchers worldwide. Within the last few years, methods based on microarray technology have been adapted to the analysis of proteins, and novel applications have emerged (for a review see Reference 24). Protein microarrays offer the fascinating possibility to study protein interactions in a massively parallel fashion, including protein-protein(26), enzyme-substrate (20,27), protein-DNA (7), protein-oligosaccharide, or protein-drug interactions $(2,19)$. The use of protein microarrays in diagnostic applications has led to the development of novel products into marketable commodities (for an overview see http://www.biochipnet.de). Meanwhile, researchers are endeavoring the application of array-based proteomic approaches to the whole proteome of a cell within a single experiment $(1,9,13)$.

A prerequisite for any array-based approach is the availability of a set of capture molecules that bind to their targets with high affinity and known specificity (5). With regard to nucleic acids, the generation of such capture molecules does not pose problems. Complementary capture molecules can easily be predicted from the primary sequence of the target and synthesized by either chemical methods or PCR amplification.

In the protein world, however, the prediction of a specific capture agent from the primary amino acid sequence is not possible. Nevertheless, methods to generate and identify capture molecules are available (11), but antibody production and characterization is still a laborious process.

Antibodies are the most prominent capture molecules. They exhibit very high affinities to their targets and show a high specificity upon binding. Other types of capture molecules include recombinant proteins generated by phage or ribosomal display techniques. Recently, a variety of other types of binders was developed (e.g., aptamers, trinectin scaffolds) that might become valuable alternatives to antibodies $(16,17)$.

Protein microarrays have also been shown to be valuable tools in the characterization of protein-protein interactions (25). They offer a very fast and attractive possibility to analyze the specificity and selectivity of capture molecules simultaneously $(8,12)$. Thus, the possibility of screening large sets of capture molecules will no doubt accelerate the identification of highly specific high affinity capture probes, which do not exhibit cross-reactivity.

A more specific prerequisite for protein microarray-based screening approaches is that the immobilized proteins must be presented in their native functional state. This can be achieved for many proteins, but is quite difficult with important proteins, such as transmembrane proteins $(4,18,26)$. Notwithstanding, transmembrane proteins, such as $\mathrm{G}$ protein-coupled receptors (GPCR), could be successfully immobilized in a micropatterned format, both with defined orientation to their substrates and retaining a functional state. Ligand binding, G protein activation, and receptor deactivation could be successfully demonstrated for bovine rhodopsin, for example (4). This approach could also be of great interest in the characterization of capture molecules, but scaling up such a system still presents a real challenge.

The optimal environment for the stabilization of cell surface proteins is the cell membrane itself. Therefore, cell microarrays 
seem to be a promising alternative in the characterization of binding molecules that are specific for cell surface antigens. Transfected cell microarrays were described recently (28). In their study, clusters of cells overexpressing defined cDNAs were immobilized on the slides as tiny spots. These cell microarrays can be used for screening for the function of gene products involved in biological processes or, after the expression of membrane receptors, for the characterization of specific capture molecules, such as antibodies.

Here, we describe a different approach in generating cell microarrays and their application in characterizing the binding of membrane protein-specific antibodies to cell surface markers. Cells with a known cell surface protein panel were used to generate the cell microarrays. Their potential use in characterizing antibodies was evaluated by employing a simple assay procedure that revealed either presence or absence of distinct cell surface proteins. Therefore, printed cell microarrays might well be used to characterize large numbers of antibodies or capture molecules with respect to their binding abilities to conformationally welldefined preserved cell surface molecules.

\section{MATERIALS AND METHODS}

\section{Cell Lines and Growth Conditions}

The human monocytic cell line THP-1 (DSMZ [Braunschweig, Germany] number ACC 16); two different human Bcell lines, Boleth Bo (ECCAC [Salisbury, Wiltshire, England] IHW number 9031) and WT-51 (IHW number 9029); the human histiocytic lymphoma cell line U937 (DSMZ number ACC 5) and the human T-cell line Jurkat (DSMZ number ACC 282) were cultivated in RMPI 1640 with $10 \%$ fetal calf serum (FCS). The human osteosarcoma cell line Saos-2 (ATCC [Manassas, VA, USA] number HTB-85) was grown in modified McCoy's 5A (BioWhittaker Europe, Vervier, Belgium) with 15\% FCS. The human neuroblastoma cells SH-SY5Y (ATCC number CRL-2266) were grown in 45\% HAM F12 and 45\% minimum essential medium (MEM) Eagle's without glutamine, 1\% MEM with unessential amino acids, and 9\% FCS. The human embryonic kidney cell line HEK293 (ATCC number CRL-1573) and the mouse fibroblast cell line 3T3-Swiss albino (ATCC number CCL-92) were grown in Dulbecco's modified Eagle medium (DMEM) high glucose with 10\% FCS. The Chinese hamster ovary cell line CHO-DUKX was grown in MEM- $\alpha$ (BioWhittaker Europe) with $10 \%$ FCS. All cell lines were grown at $37^{\circ} \mathrm{C}$ at $5 \% \mathrm{CO}_{2}$. The rat adrenal gland cell line PC12 was grown in 82.5\% Hank's F12K, 15\% horse serum (PAA Laboratories, Cölbe, Germany), and $2.5 \% \mathrm{FCS}$ at $37^{\circ} \mathrm{C}$ in $10 \% \mathrm{CO}_{2}$.

All media contained $2 \mathrm{mM}$ glutamine and $100 \mathrm{U} / \mathrm{mL}$ of penicillin-streptomycin. Adherent cells were briefly detached by trypsin-EDTA (Roche Diagnostics GmbH, Mannheim, Germany) before harvesting. All reagents were purchased from GibcoBRL, Eggenstein, Germany, if not specified otherwise.

\section{Antibodies}

The following antibodies were used: mouse monoclonal antihuman IgG antibodies (mAbs); major histocompatibility complex (MHC)-specific antibodies L243 (anti-human leukocyte antigen [HLA]-DR) (15); TÜ 36 (anti-HLA-DR; Pharmingen, San Diego, USA); TÜ 39 (anti-HLA-DR, -DP, and most -DQ; a gift from Prof. C. Müller, University of Tübingen, Germany) (29); W6/32 (anti-HLA-A, -B, -C; Biotrend, Cologne, Germany) (3); GAP A3 (anti-HLA-A3); BB7.2 (anti-HLA-A2); B1.23.2 (anti-HLA-B, -C) (6); anti-CD 3 (CBL-150; Cymbus Biotechnology Ltd., Chandlers Ford, England) (10); anti-CD 45 (clone EO1; Diatec, Oslo, Norway); anti-CD 45 (clone 69; BD Biosciences, San Jose, USA); anti-epidermal growth factor receptor (EGFR) mAb10 and mAb11 (all NeoMarkers, Newmarket, England). $\mathrm{Cy}^{\mathrm{TM}} 3$-conjugated goat anti-mouse IgG plus IgM (Dianova, Hamburg, Germany) were used as detection antibodies at a concentration of $0.75 \mu \mathrm{g} / \mathrm{mL}$.

\section{Generation of Cell Microarrays}

Approximately $10^{7}$ cells were harvested by centrifugation ( 5 minutes, $400 \times g)$ and the supernatant was carefully removed. The cells were gently resuspended in the remaining liquid (approximately $50 \mu \mathrm{l}$ ). For storage purposes, these cells can be fixed with $0.5 \%$ paraformaldehyde (PFA) (Merck, Darmstadt, Germany) in at least $1 \mathrm{~mL}$ phosphate-buffered saline (PBS) on ice for 30 minutes. Subsequently, the cells were washed; the cell pellet was kept in $10 \mathrm{~mL}$ of PBS and could thus be stored at $4^{\circ} \mathrm{C}$ in the dark for at least 10 days before printing the cell microarrays.

Fifty microliters of different cell suspensions were used directly for the generation of the cell arrays. Depending on cell type and cell number, Biocoll (Biochrom AG, Berlin, Germany) was added to the cells. Biocoll preserves a homogenous cell suspension by preventing the sedimentation of the cells onto the bottom of the printing reservoir. Two microliters of $80 \%$ glycerol in PBS were added to $50 \mu \mathrm{L}$ cell suspension in order to prevent total dehydration after the printing process.

A GMS Microarrayer 417 (pin- and ring-system; MWGBIOTECH AG, Ebersberg, Germany) was used to transfer cells onto poly-L-lysine-coated poly-prep slides (Sigma, Taufkirchen, Germany). Approximately $50 \mathrm{pL}$ were transferred to the glass surface by a single printing event. To examine transfer efficiency and reproducibility of the printing process, test arrays of 400 replicates $(20 \times 20$ spots $)$ were generated in a single run.

After the printing process, the cell microarrays were incubated for 1 hour at $37^{\circ} \mathrm{C}$ to ensure the best possible ionostatic interaction between the cells and the poly-L-lysine surface of the slides. The cells were fixed by incubating the entire array in a drop of $10 \%$ PFA in PBS for 10 minutes at room temperature. This fixation step was omitted in arrays, which consisted only of cells that had been fixed directly after harvesting.

To allow the incubation of the cell arrays in a defined volume, they were framed using a hydrophobic pen, (PAP Pen; SCI Science Service, Munich, Germany). By adding $30 \mu \mathrm{L}$ of the blocking solution 1.5\% BSA Fraction V (Carl Roth, Karlsruhe, Germany), 5\% skim milk powder (PAA Laboratories) in PBS for 45 minutes at room temperature, the glass surface was loaded with protein to minimize nonspecific protein adsorption during the assay. The arrays could then be used directly for the assays or be stored in the dark at room temperature after drying. Stored cell microarrays were rehydrated by incubating the slides with PBS for 5 minutes and by three 5 -minute incubation steps with the washing buffer $0.1 \%$ Tween ${ }^{\circledR} 20$ (Merck) in PBS. 


\section{Assay Procedure and Immunofluorescence}

Standard immunofluorescence protocols were adapted to the microarray-based procedure. For the staining of printed cell arrays, different dilutions of primary antibodies in assay buffer (1.5\% BSA Fraction V, 2.5\% skim milk powder, $0.1 \%$ Tween 20 in PBS) were tested; all antibody incubations were performed in a humid chamber for 45 minutes. After each antibody incubation step, the cell microarrays were washed 4 times (for 1 minute) with $0.1 \%$ Tween 20 in PBS followed by an additional washing step (for 5 minutes) with $0.1 \%$ Tween 20 in PBS. Cy3conjugated goat anti-mouse IgG plus IgM (Dianova) were used as detection antibodies at a concentration of $0.75 \mu \mathrm{g} / \mathrm{mL}$ for 45 minutes at room temperature. The described washing procedure was repeated, and the processed cell microarrays were washed in cool water for a few seconds before rinsing them with deionized water. All slides were carefully dried in a stream of nitrogen.

\section{Laser Scanning and Fluorescence Microscopy}

Pictures of the stained cell microarrays were taken using a confocal laser fluorescence scanner (Array-Scanner GMS 418; MWG-BIOTECH AG) at maximum laser power at highest resolution. For quantification purposes, the gain was set to $20 \%$. Cell microarrays were quantified based on the median of the fluorescence intensities of each spot using the ImaGene 4.0 software package (BioDiscovery, Marina Del Rey, CA, USA).

For visualization of cell nuclei, the microarrays were incubated with Hoechst 33258 (Sigma) diluted 1:1000 for 5 minutes at room temperature. After 3 washes with deionized water, the pictures of the stained arrays were taken using an Axiophot fluorescence microscope (Carl Zeiss, Jena, Germany) (excitation: 365 $\mathrm{nm}$; emission of $478 \mathrm{~nm}$ ) equipped with a charge-coupled device (CCD) camera (Coolpix 990; Nikon GmbH, Düsseldorf, Germany). Before printout, the images were modified in brightness, contrast, and intensity using Corel PHOTO-PAINT 8 (Corel Corporation, Ottawa, Canada).

\section{RESULTS AND DISCUSSION}

Microarrays, consisting of a number of defined cell types, could prove to be a powerful tool to characterize binding events on cell surface receptors. Major advantages are the parallelized and miniaturized assay format and the possibility to detect binding to receptors that are present in a native environment. In a first approach to evaluate such a system, we developed a method that allowed the parallel characterization of antibody binding using cell arrays. It was important to develop a method that takes advantage of commercially available technology (i.e., arrayers and scanners for DNA and protein microarray applications) and that can be easily established in laboratories possessing an array facility.

In a first step towards the production of cell microarrays, a robust protocol for cell printing was established. Using a contact-printing without any technical modifications, conditions for the efficient transfer of cells were examined. To generate spots consisting of a similar number of cells, different buffer systems were tested. Suspensions of cells were prepared at a very high cell density $\left(10^{9}\right.$ cells $\left./ \mathrm{mL}\right)$ and used directly for printing.
This method proved to be efficient for cells that could be grown at very high density. Thus, a large number of cells can be transferred, and reproducible spots can be generated even under difficult conditions (e.g., during long printing processes [> 1hour]) when cell sedimentation is observed in the printing reservoir. When the suspensions contained fewer cells, it was necessary to add substances such as Biocoll or glycerol in order to adjust the density of the printing solution to the same density as the cells and thus prevent sedimentation.

An optimized printing solution is important for the generation of uniform spots and also for the preservation of protein structures in the cell membrane. The addition of glycerol to the printing buffer prevents the total evaporation of water from the tiny cell-containing droplets that are deposited during printing. Therefore, cells are kept within a hydrated microenvironment during the whole immobilization process and even during longterm storage of the microarrays. The addition of glycerol also helps to conserve native protein structures in the cell membrane and, thus, the antigenicity of the cell surface molecules.

Since the cell arrays are generated by the mechanical contact of a steel pin with a solid glass support, the influence of the printing process on shape and integrity of the printed cells was analyzed. The number of cells destroyed by micromechanical shear forces during cell deposition was regarded as perfectly acceptable, as long as there was no more than a single surface contact of the needle. Increasing the number of needle contacts led to an increase in the total number of transferred cells, but also to a significant mechanical damage of the printed cells and, thus, to a greater background signal during the assay.

To examine the efficiency and uniformity of the printing process, experiments using the human monocytic cell line THP1 were performed. Cells were transferred with a single hit of the pin onto each microspot using an adjusted cell concentration of $10^{9}$ cells $/ \mathrm{mL}$ in the printing reservoir. Cells were deposited on a positively charged substrate (poly-L-lysine-coated glass slides), and immobilization was achieved via electrostatic interactions. Arrays consisting of $20 \times 20$ spots were created, and a specific cell surface marker, the lymphocyte common antigen (LCA), was detected after immunofluorescence staining with an antiCD $45 \mathrm{mAb}$. In addition, the nuclei of the cells were stained.

A typical scan image is depicted in Figure $1 \mathrm{~A}$ and shows a homogeneous spot pattern for the 400 spot array. In addition, enlarged microscope images of stained nuclei in single microspots (Figure 1, B, D, and F) reveal the localization of the individual cell; the fluorescent signals caused by immunostaining elucidate the distribution of LCA.

The picture of the stained nuclei was used to count the cells and determine the cell number per spot for the entire array. The average cell number in a microspot was 18 , the standard deviation 4.9 (Figure $1 \mathrm{H}$ ). The individual cell numbers ranged from 5 up to 38 cells with $80 \%$ of all microspots containing $25 \pm 10$ cells. Only $20 \%$ of the microspots contained less than 15 or more than 35 cells per spot; at least $95 \%$ of all microspots were loaded with more than 10 cells (Figure $1 \mathrm{H})$. Since fewer than $5 \%$ of the microspots contained 10 cells per spot or less, the probability of having two spots with less than 10 cells per spot is well below $1 \%$. Therefore, the probability of obtaining at least two spots with more than 10 cells is high enough when each cell type sample is printed in triplicate. This is important for the setup of the robust 


\section{RESEARCH REPORT}

cell microarray-based assay described below. These data demonstrate that it is possible to immobilize cells in a reproducible manner in a microarray format using standard arraying equipment.

When this type of cell microarray is employed as a simple screening tool, one must make certain that the assay procedure is robust enough to cope with the statistical variations of the cell numbers. Therefore, the number of cells that is required in the printing solution to exhibit a significant signal in an immunoassay was determined. Three different cell lines (human T-cell line Jurkat, human embryonic kidney cell-line HEK293, and hu-
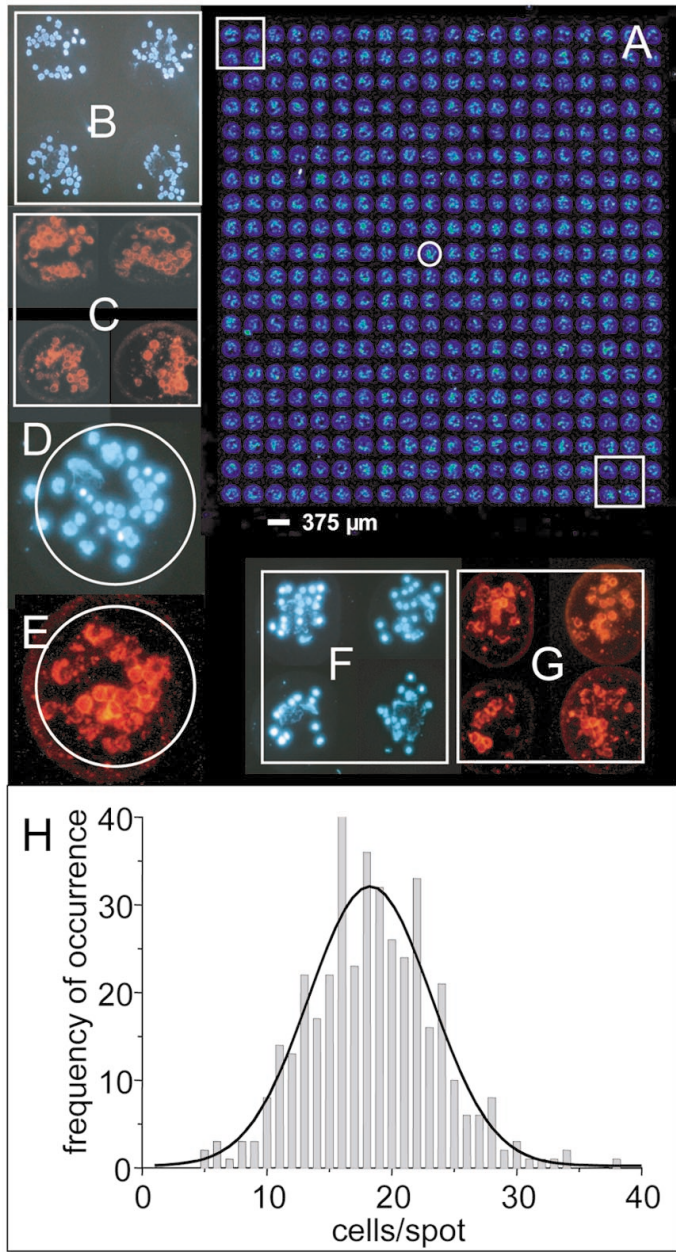

Figure 1. Generation of cell microarrays. A $20 \times 20$ spot array was generated from a suspension containing THP-1 cells. The 400 cell spots were printed in a single run using the GMS417 contact printer. To visualize the nuclei of the deposited cells, a standard DNA staining protocol was employed. The LCA present on the surface of THP-1 was detected by immunostaining with an anti-CD 45 antibody. A fluorescence image of the whole array was created using a standard microarray scanner (GMS418) at the highest resolution of $10 \mu \mathrm{m}$. Details of the array were photographed using an Axiophot fluorescence microscope. (A) Shows a picture of the complete array. The equal distribution of signal intensities over the 400 cell microspots is indicated by similar colors in the chosen pseudocolor presentation. Framed spots are also shown in detail; microscopic images of dyed nuclei are seen in panels $\mathrm{B}, \mathrm{D}$, and $\mathrm{F}$, and the corresponding immunostain is shown in panels $\mathrm{C}, \mathrm{E}$, and $\mathrm{G}$. The diagram $(\mathrm{H})$ shows a frequency distribution of cell numbers per spot numbers. A Gauss fit of the normally distributed data resulted in an average of 18.3 cells/spot and a standard deviation of 4.9 cells/spot. man monocytic cell line THP-1) were diluted in printing buffer containing Biocoll (5 different cell densities ranging from $10^{8}-10^{9}$ cells $/ \mathrm{mL}$ ) and immobilized in triplicate in an arrayed format. The generated arrays were visualized by staining the nuclei and photographed (Figure 2, A-C, row 1). CD45 was chosen as specific cell surface marker and detected by immunostaining with the anti-CD45 (Figure 2, A-C, row 2). Nucleus staining revealed that all spots contained cells. Immunofluorescent signals were observed for the CD45-positive Jurkat (A) and THP-1 (C) cells, whereas the CD45-negative HEK293 cells gave no signal. Spots generated from dense cell suspensions contained more cells, which also appeared to be more homogeneously distributed (Figure 2, upper row). The influence of cell density on signal intensity derived from immunostaining is also depicted in Figure 2. The signal was quantified from an image generated by a GMS418 biochip scanner and the median was calculated for each spot by the ImaGene4.0 software.

Only when the printing buffer contained a large number of cells $(80 \%$ or $100 \%$ of maximum cell density), could a significant and stable signal be measured. Therefore, it is recommended that cells always be spotted at the highest possible concentration in order to guarantee a sufficient number and homogeneous distribution of cells in a microspot. The final cell array was generated from 11 different cell lines. Antibodies with known specificity against human MHC class I (W6/32) and human MHC class II (L243) were used as ligands, and interaction was detected using an immunofluorescence procedure. MHC class I molecules are known

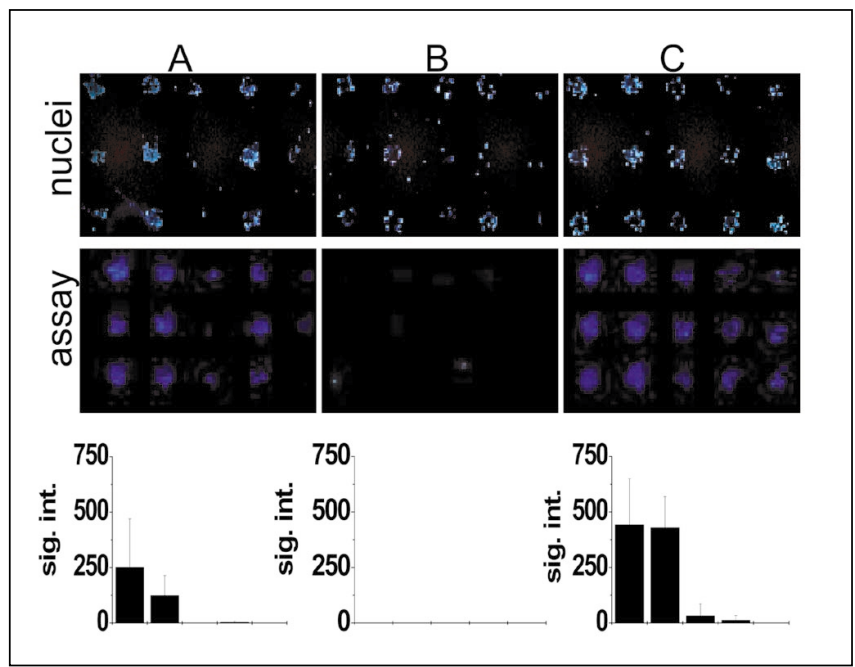

Figure 2: Influence of cell density on the generation of cell microarrays. Three cell lines (A) Jurkat, (B) HEK293, and (C) THP-1 were printed in an arrayed format to examine the effect of different cell densities in the printing solution on assay performance. For each cell type, a cell suspension $\left(10^{9} \mathrm{cell} / \mathrm{mL}\right)$ was mixed with increasing volumes of a Biocoll solution $(0 \%, 20 \%, 40 \%, 60 \%$, and $80 \%)$; triplicates from these dilutions were used to create cell spots in triplicate. The created arrays were subjected to DNA staining (top row) and immunostaining with anti-CD45 antibody (second row). A pseudocolor representation of images from the immunostained cells taken with a biochip array scanner was chosen for visualizing the spots. Both leukocytic cell lines ( $A$ and $C$ ) gave specific signals for CD45, while microspots consisting of HEK293 cells (B) (CD45 negative) showed no stained cells at all. The graphs below the images show the mean of the signal intensities as determined for the individual spots (see Materials and Methods). The determined values show that a high density of cells in the printing solution is required to generate cell microarrays. 
to be present on all human cell lines, but their surface density can, however, differ significantly between different cell types and cell lines due to differences in cell surface expression. In contrast, MHC class II molecules are only expressed on a subpopulation of hemopoetic cells. Class II molecules are highly expressed on B cells and antigen-presenting cells, but only to a moderate degree on T cells (23). To illustrate the flexibility of the established arraying protocol, different cell suspensions or adherent cell lines were used for the experiments; B cells (Boleth Bo, WT-51) and monocytic cell lines (THP-1) were grown in suspension cell cultures, whereas fibroblast (3T3) or neuroblastoma cells (SH-SY5Y) were grown as adherent cell lines. Adherent cell lines were harvested and printed after a small period of trypsination. This procedure does not reduce the ability of antibodies to recognize MHC antigens (21). Cell microarrays were immunostained with MHC Ispecific (Figure 3, A-E) and MHC II-specific (Figure 3, F-J) antibodies, and signal intensities were calculated for each triplicate of microspots of the same cell line. Cells expressing high amounts of MHC class I or MHC class II receptors were easily identified by their respective high affinity anti-MHC I antibodies W6/32 and anti-MHC II antibodies L243. The differences in signal intensities observed for the different cell lines expressing the desired MHC molecules correlate with known differences in expression rates for the receptors in the different cell types.

This setup was used to internally calibrate the assay system. Cells, which did not possess the surface antigen or only expressed it at very low levels, did not lead to any signal or only to a very weak one. The nonhuman cell lines (3T3-S, CHO) showed neither MHC I- nor MHC II-specific significant antibody binding. The observed background signal is most likely caused by scattered light from the immobilized cells. The PC-12 rat cell line, which presents neither MHC I nor MHC II, revealed a significantly high background for either antigen. This background was found for PC-12 cells even after incubation of a secondary detection antibody (data not shown), whereas all other printed cell lines produced no signals at all. The B cell lines Boleth Bo and WT-51 showed very strong MHC II-specific antibody binding, whereas the THP-1 monocytes, the U937 histiocytes, and the Jurkat T-cells revealed only moderate binding. MHC II negative human cell lines HEK293 and Saos-2 showed no binding of the MHC II-specific antibody, whereas the SHSY5Y neuroblastoma cell line revealed some background signals. MHC I was detected as a strong to moderate signal in all human cell lines, whereas nonhuman cell lines, except for nonspecific PC-12 binding, produced no signals. Our data, thus, demonstrate that highly expressed receptors can be detected in a reliable manner; cell lines, which only express a small number of cell surface antigens, are not easily distinguished from negative cells.

To compare the fluorescent intensities detected by fluorescence microscopy with those obtained using the biochip scanner, microscopic images were taken from two selected spots of the cell arrays. The images of transmission microscopy of microspots containing WT-51 (Figure 3B) and THP-1 (Figure $3 \mathrm{G}$ ) cells corresponded to the images of the stained nuclei (Figure 3, $\mathrm{C}$ and $\mathrm{H}$ ). Cells were well distributed and could be counted easily. The microscopic images of immunostained MHC II (Figure 3D) and MHC I cells (Figure 3I) had a better resolution than spots acquired with the biochip reader (Figure 3, E and J, pseudocolor-fluorescence image). Due to the $10-\mu \mathrm{m}$ optical res- olution of the scanner, each single cell was depicted by approximately 4 pixels. In our system, this resolution was sufficient to discriminate between a strong positive interaction and a negative or weak interaction of the antibody with the cell surface. The fairly large standard deviations in median fluorescence signal intensities (Figure 3, diagram) resulted from the differences

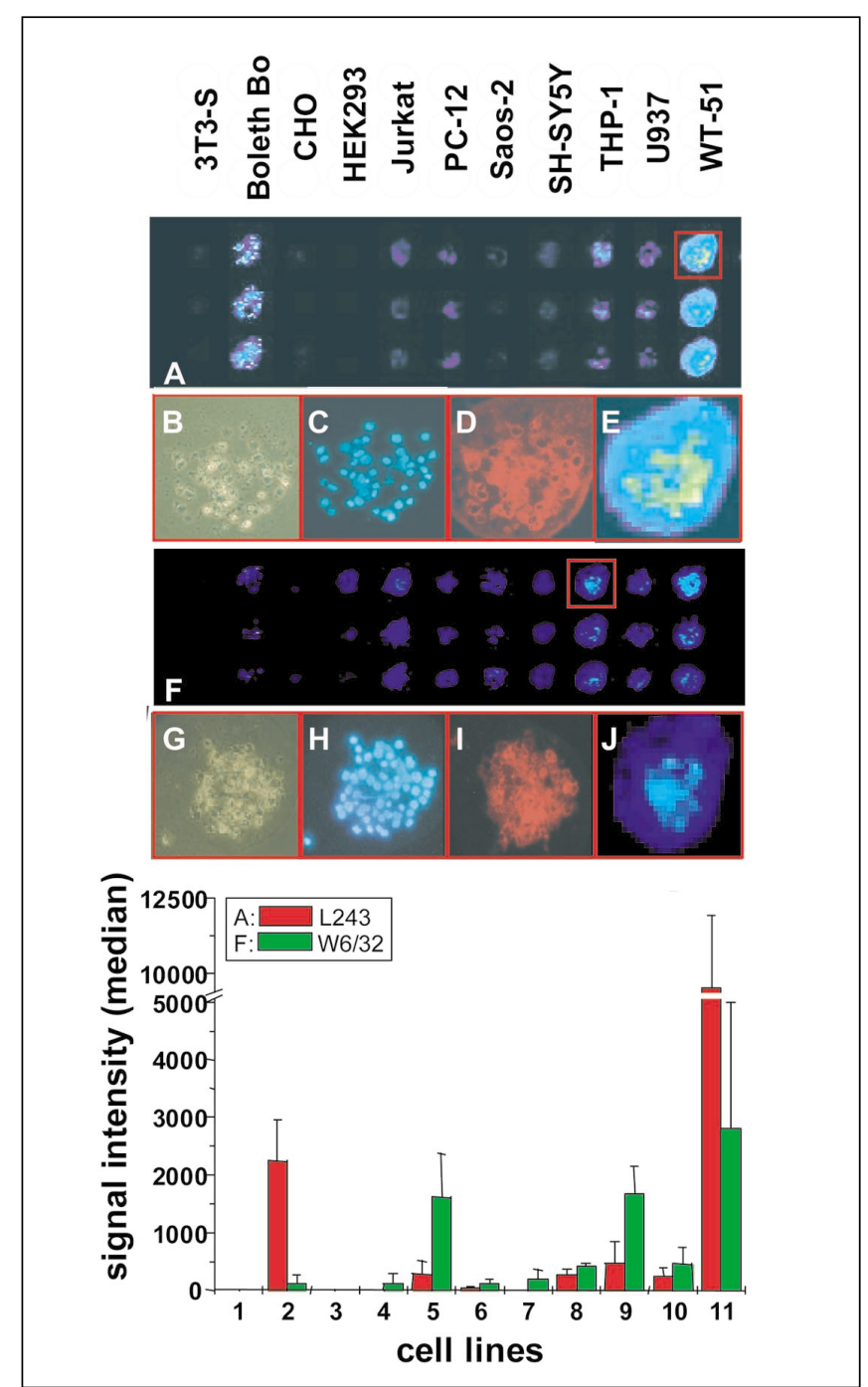

Figure 3. Close-up view on cell microarrays-fluorescence microscopy detection compared to biochip scanner imaging. Arrays of cells were printed using 11 different cell lines; the location of the different cells is indicated in the top row. These microarrays were used to detect MHC class I and class II cell surface antigens on the different cell lines using the developed immunofluorescence staining method. The first set of images (A-E) was taken from arrays stained with a monoclonal antibody specific for MHC class II (L243); images (F-K) show pictures from arrays stained with the MHC class I antibody W6/32. Pictures in panels A, E, F, and J were acquired with a biochip scanner and are shown in pseudocolor, the other pictures are micrographs taken with a fluorescence microscope. Framed spots from panels A and F are shown in detail at higher magnification. Images in panels B and $\mathrm{H}$ were taken by transmission microscopy; panels $\mathrm{C}$ and I show the same spots after a DNA staining procedure; panel E shows the specific MHC class II signal, and panel J shows the MHC class I signal. The signals for the different cell lines were quantified, and the results for the 11 cell lines are shown in the bar plot. Strongly expressed cell surface antigens (MHC I in Jurkat, THP-1, WT-51; MHC II in Boleth Bo, WT-51) gave strong signals, whereas receptors expressed at low level produced only weak signals, which resembled those of nonspecific background. 
in cell numbers per microspot. Therefore, this system does allow only the rapid characterization of the specificity of different binding molecules against strongly expressed cell surface antigens, but not the fine discrimination of different amounts of cell surface molecules on different cell lines.

In a final experiment, 12 different antibodies were screened for selectivity and specificity against cell surface markers using a cell microarray of 10 different adherent and nonadherent cell lines. After incubation with secondary detection antibody only

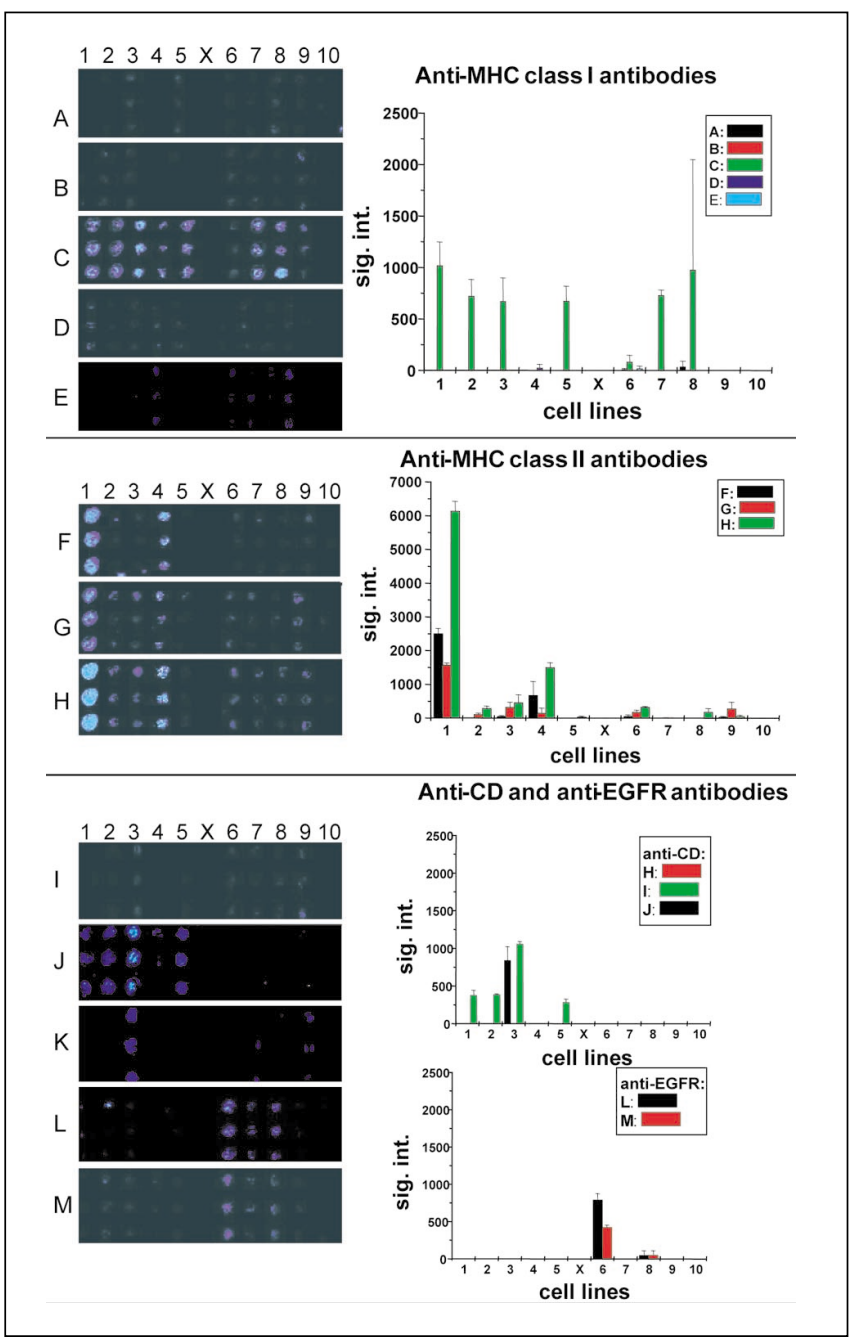

Figure 4. Antibody characterization using cell microarrays. Ten different cell lines were printed in triplicate in an arrayed format for the antibody characterization experiment. The immobilized cell types are: (1) WT-51; (2) THP-1; (3) Jurkat; (4) Boleth Bo; (5) U937; (X) negative control (6) SH-SY5Y; (7) Saos-2; (8) HEK293; (9) 3T3; and (10) CHO. Twelve different monoclonal antibodies with known specificity for MHC class I, MHC class II, CD45, CD3, and EGFR were tested. Assays were performed according to the described protocol using a Cy3-labeled secondary antibody. Scanned images of the arrays used with the different antibodies are shown. The mean signal intensity obtained with the different cell types is shown in the bar charts. The first diagram shows the result for four known antiMHC class I antibodies: (A) GAP A3, (B) BB7, (C) W6/32; (D) B1.23.1, and (E) negative control. The second diagram shows the result for three known anti-MHC class II antibodies: (F) L243, (G) TÜ36, and (H) TÜ39. The first diagram shows two anti-CD45 antibodies: (I) clone 69, (J) EO, and one anti-CD3-specific antibody (K) (CBL-150). Reactivity of anti-EGFR (mAb10) (L) and anti-EGFR $(\mathrm{mAb} 11)(\mathrm{L})$ is shown in the fourth diagram. The results are discussed in the text.
(Figure 4E), either no signals or very weak nonspecific background signals were detected, which however did not interfere with the strong specific signals of the assays.

First, printed cells were analyzed for the presence of MHC class I receptors. Four different MHC I-specific antibodies were incubated on the cell microarrays: $\mathrm{mAb}$ W6/32 (Figure 4C), which recognizes all HLA-A, $-B$, and $-C$ gene products; $m A b$ B1.23.2, which recognizes anti-HLA-B, -C, anti-HLA-A3 mAb GAP A3, and anti-HLA-A2 mAb BB7.2.

The MHC I-specific mAb W6/32 gave a strong signal for 6 of the 8 human cell lines, indicating that these cells strongly expressed HLA-A antigens on their surface. SH-SY5Y cells exhibited a low signal for the MHC class I receptors, whereas no significant signals for any MHC class I molecule could be detected on the surface of the Boleth cell line (Figure 4, A-D).

Immunostaining of the cell microarrays with the MHC class II-specific antibodies mAb L243 (anti-HLA-DR), mAb TÜ 36 (anti-HLA-DR), and mAb TÜ 39 (anti-HLA-DR, -DP, and most -DQ) produced signals for all MHC-II positive cell lines. However, only the moderate signal for mAb TÜ 39 and $\mathrm{mAB}$ L243 on Boleth Bo and the strong signals for the three antiMHC II antibodies on WT-51 were significant. The strongest signal was detected for WT-51. Two antibodies, Tue 36 and Tue 39 , revealed some nonspecific background signals with nonMHC II-presenting cell lines (SH-SY5Y, HEK293, 3T3), whereas $\mathrm{L} 243$ produced no nonspecific background signals or cross-reactivity with non-MHC II cells at all. This shows that cell arrays can be used as a tool for screening for selectivity and specificity, but also points to the limitations of the current system.

Antibody screening for the specific binding to other cell surface antigens was carried out using anti-CD45, anti-CD3, and anti-EGFR binders. The $\mathrm{T}$ cell-specific antibody $\mathrm{mAb}$ CBL-150 (anti-CD3) only reacted with the T cell line Jurkat (Figure 4K) in a very specific manner. Anti-CD $45 \mathrm{mAb}$ EO1 recognized the B cell lines WT-51, THP-1, and U937 and T- cell line Jurkat (Figure 4J) without an indication of any cross-reactivity or nonspecific binding to other cell surface structures. In contrast, another CD45-specific antibody (mAb clone 69) did not bind to any of the CD45 positive cells (Figure 4I).

Immunostaining with the two EGFR-specific antibodies, $\mathrm{mAb} 10$ and $\mathrm{mAb} 11$, led to significant signals on SH-SY5Y and HEK 293 cells (Figure 4, L and M). The signals of both antibodies were much stronger for SH-SY5Y, which would thus be the better model cell line for an EGFR antibody screen.

\section{CONCLUSION}

These results demonstrate that cell microarrays can be generated easily using standard arraying equipment. The generated cell arrays were used as a screening tool for the characterization of cell surface-specific antibodies. Different antibodies were incubated on a variety of immobilized cell lines, and information on selectivity and specificity against cell surface antigens was acquired in a simple assay. While the quality of the established system only allows the discrimination binding versus nonbinding, advances in assay performance will help to make cell arrays useful tools in the detailed profiling of surface receptor expression. The possibility to set up highly parallel systems that will allow 
the detection of reactivity and cross-reactivity of a given antibody with a whole collection of cell lines within the same experiment will speed up the screening capacity for specific antibodies. Through their miniaturization, cell microarrays are quite economical, since only small quantities of antibodies are needed.

As an alternative to the printing approach described in this manuscript, cell microarrays can be generated using the "living microarray technology" (28). One limitation of this approach, however, is intrinsic to the process of transfection. Because of their very high transfection efficiencies, only cell lines such as HEK or COS proved to be compatible with this technology. This makes most primary cell lines, which can have transfection efficiencies of less than $1 \%$ unlikely candidates for use in such microarray formats.

In contrast to living microarrays, printed cell microarrays allow the immobilization of any type of cell line. All cells expressing the antigen of interest at a significant level will lead to a positive signal, whereas cells without the antigen of interest or with low antigen expression will exhibit no or only weak signals. Cross-reactivity or nonspecific binding of tested antibodies results in signals derived from immunostained control cells. This helps to sort out cross-reactive binders at an early stage and, thus, enables the fast identification of optimal antibodies.

In the context of array-based proteomics, enormous efforts are undertaken to generate the large numbers of capture molecules required for this approach. Screening tools to identify capture molecules in a fast and, therefore, economic way are required. Membrane proteins are notoriously difficult to handle, and the immobilization of functional proteins on protein arrays is a real challenge. Thus, a way to present membrane proteins to identify binders is to retain them within their native environment-on cellular microarrays.

\section{ACKNOWLEDGMENTS}

We would like to thank Stefan Stevanovic, Hubert Kalbacher, and Oliver Pötz for antibodies, cell lines, and many fruitful discussions. The critical reading of the manuscript by Jutta Bachmann is gratefully acknowledged.

\section{REFERENCES}

1.Albala, J.S. and I. Humphrey-Smith. 1999. Array-based proteomics: highthroughput expression and purification of IMAGE consortium cDNA clones. Curr. Opin. Mol. Ther. 1:680-684.

2.Arenkov, P., A. Kukhtin, A. Gemmell, S. Voloshchuk, V. Chupeeva, and A. Mirzabekov. 2000. Protein microchips: use for immunoassay and enzymatic reactions. Anal. Biochem. 278:123-131.

3.Berger, A.E., J.E. Davis, and P. Cresswell. 1982. Monoclonal antibody to HLA-A3. Hybridoma 1:87-90.

4.Bieri, C., O.P. Ernst, S. Heyse, K.P. Hofmann, and H. Vogel. 1999. Micropatterned immobilization of a $\mathrm{G}$ protein-coupled receptor and direct detection of $\mathrm{G}$ protein activation. Nat. Biotechnol. 17:1105-1108.

5.Borrebaeck, C.A. 2000. Antibodies in diagnostics-from immunoassays to protein chips. Immunol. Today 21:379-382.

6.Breban, M., J.L. Fernandez-Sueiro, J.A. Richardson, R.R. Hadavand, S.D. Maika, R.E. Hammer, and J.D. Taurog. 1996. T cells, but not thymic exposure to HLA-B27, are required for the inflammatory disease of HLA-B27 transgenic rats. J. Immunol. 156:794-803.

7.Bulyk, M.L., E. Gentalen, D.J. Lockhart, and G.M. Church. 1999. Quantifying DNA-protein interactions by double-stranded DNA arrays. Nat. Biotechnol. 17:573-577.
8.Bussow, K., D. Cahill, W. Nietfeld, D. Bancroft, E. Scherzinger, H. Lehrach, and G. Walter. 1998. A method for global protein expression and antibody screening on high-density filters of an arrayed cDNA library. $\mathrm{Nu}-$ cleic Acids Res. 26:5007-5008.

9.Cahill, D.J. 2000. Protein arrays: a high-throughput solution for proteomics research? Proteomics: A Trends Guide. 47-51.

10.Campana, D., J.S. Thompson, P. Amlot, S. Brown, and G. Janossy. 1987. The cytoplasmic expression of CD3 antigens in normal and malignant cells of the T lymphoid lineage. J. Immunol. 138:648-655.

11.Clackson, T., H.R. Hoogenboom, A.D. Griffiths, and G. Winter. 1991. Making antibody fragments using phage display libraries. Nature 352:624628.

12.de Wildt, R.M., C.R. Mundy, B.D. Gorick, and I.M. Tomlinson. 2000. Antibody arrays for high-throughput screening of antibody-antigen interactions. Nat. Biotechnol. 18:989-994.

13.Emili, A.Q. and G. Cagney. 2000. Large-scale functional analysis using peptide or protein arrays. Nat. Biotechnol. 18:393-397.

14.Fields, S., Y. Kohara, and D.J. Lockhart. 1999. Functional genomics. Proc. Natl. Acad. Sci. USA 96:8825-8826.

15.Fong, S., C.D. Tsoukas, J.L. Pasquali, R.I. Fox, J.E. Rose, D. Raiklen, D.A. Carson, and J.H. Vaughan. 1981. Fractionation of human lymphocyte subpopulations on immunoglobulin coated Petri dishes. J. Immunol. Methods 44:171-182.

16.Green, L.S., C. Bell, and N. Janjic. 2001. Aptamers as reagents for highthroughput screening. BioTechniques 30:1094-1111.

17.Kreider, B.L. 2000. PROfusion: genetically tagged proteins for functional proteomics and beyond. Med. Res. Rev. 20:212-215.

18.Lueking, A., M. Horn, H. Eickhoff, K. Bussow, H. Lehrach, and G. Walter. 1999. Protein microarrays for gene expression and antibody screening. Anal. Biochem. 270:103-111.

19.MacBeath, G., A. Koehler, and S. Schreiber. 1999. Printing small molecules as microarrays and detecting protein-ligand interactions en masse. J. Am. Chem. Soc. 121:7967-7968.

20.MacBeath, G. and S.L. Schreiber. 2000. Printing proteins as microarrays for high-throughput function determination. Science 289:1760-1763.

21.Marshall, J.F., D.C. Rutherford, A.C. McCartney, F. Mitjans, S.L. Goodman, and I.R. Hart. 1995. Alpha v beta 1 is a receptor for vitronectin and fibrinogen, and acts with alpha 5 beta 1 to mediate spreading on fibronectin. J. Cell Sci. 108:1227-1238.

22.Phimister, B. 1999. The chipping forecast. Nat. Genet.

23.Rammensee, H., J. Bachmann, and S. Stevanovic. 1997. MHC Ligands and Peptide Motifs. Landes Bioscience, Georgetown.

24.Templin, M.F., D. Stoll, M. Schrenk, P.C. Traub, C.F. Vohringer, and T.O. Joos. 2002. Protein microarray technology. Trends Biotechnol. 20:160-166.

25.Walter, G., K. Bussow, D. Cahill, A. Lueking, and H. Lehrach. 2000. Protein arrays for gene expression and molecular interaction screening. Curr. Opin. Microbiol. 3:298-302.

26.Zhu, H., M. Bilgin, R. Bangham, D. Hall, A. Casamayor, P. Bertone, N. Lan, R. Jansen, et al. 2001. Global analysis of protein activities using proteome chips. Science 293:2101-2105.

27.Zhu, H., J.F. Klemic, S. Chang, P. Bertone, A. Casamayor, K.G. Klemic, D. Smith, M. Gerstein, M.A. Reed, and M. Snyder. 2000. Analysis of yeast protein kinases using protein chips. Nat. Genet. 26:283-289.

28.Ziauddin, J. and D.M. Sabatini. 2001. Microarrays of cells expressing defined cDNAs. Nature 411:107-110.

29.Ziegler, A., J. Heinig, C. Muller, H. Gotz, F.P. Thinnes, B. UchanskaZiegler, and P. Wernet. 1986. Analysis by sequential immunoprecipitations of the specificities of the monoclonal antibodies TU22,34,35,36,37,39,43, 58 and YD1/63.HLK directed against human HLA class II antigens. Immunobiology 171:77-92.

\section{Address correspondence to:}

Dr. Thomas O. Joos

NMI-Natural and Medical Sciences Institute at the University of Tübingen

Markwiesenstr. 55

72770 Reutlingen, Germany

e-mail:joos@nmi.de 